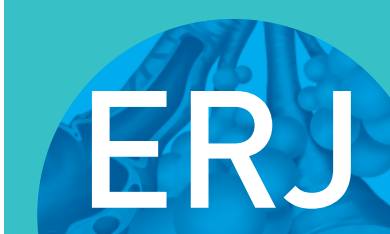

open research

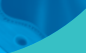

\title{
Integrating Comprehensive Geriatric Assessment for people with COPD and frailty starting pulmonary rehabilitation: the Breathe Plus feasibility trial protocol
}

\author{
Lisa Jane Brighton (10) ${ }^{1}$, Catherine J. Evans (10 ${ }^{1,2}$, Morag Farquhar (103), \\ Katherine Bristowe (10), Aleksandra Kata ${ }^{4}$, Jade Higman ${ }^{4}$, Margaret Ogden $^{5}$, \\ Claire Nolan 6,7 , Deokhee Yi (1) 1 , Wei Gao (10), Maria Koulopoulou ${ }^{8}$, \\ Sharmeen Hasan ${ }^{9}$, Claire J. Steves (10 ${ }^{10,11}$, William D-C. Man $\mathbb{1}^{6,7,12}$ and \\ Matthew Maddocks (1) 1
}

ABSTRACT One in five people with COPD also lives with frailty. People living with both COPD and frailty are at increased risk of poorer health and outcomes, and face challenges to completing pulmonary rehabilitation. Integrated approaches that are adapted to the additional context of frailty are required.

The aim of the present study is to determine the feasibility of conducting a randomised controlled trial of an integrated Comprehensive Geriatric Assessment for people with COPD and frailty starting pulmonary rehabilitation.

This is a multicentre, mixed-methods, assessor-blinded, randomised, parallel group, controlled feasibility trial ("Breathe Plus"; ISRCTN13051922). We aim to recruit 60 people aged $\geqslant 50$ with both COPD and frailty referred for pulmonary rehabilitation. Participants will be randomised 1:1 to receive usual pulmonary rehabilitation, or pulmonary rehabilitation with an additional Comprehensive Geriatric Assessment. Outcomes (physical, psycho-social and service use) will be measured at baseline, 90 days and 180 days. We will also collect service and trial process data, and conduct qualitative interviews with a subgroup of participants and staff. We will undertake descriptive analysis of quantitative feasibility outcomes (recruitment, retention, missing data, blinding, contamination, fidelity), and framework analysis of qualitative feasibility outcomes (intervention acceptability and theory, outcome acceptability). Recommendations on progression to a full trial will comprise integration of quantitative and qualitative data, with input from relevant stakeholders. This study has been approved by a UK Research Ethics Committee (ref.: 19/LO/1402).

This protocol describes the first study testing the feasibility of integrating a Comprehensive Geriatric Assessment alongside pulmonary rehabilitation, and testing this intervention within a mixed-methods randomised controlled trial.

@ERSpublications

This protocol describes the first study testing the feasibility of integrating a Comprehensive Geriatric Assessment alongside pulmonary rehabilitation for people with both COPD and frailty, and the appropriate trial methods to test its effectiveness https://bit.ly/39lZ7f1

Cite this article as: Brighton LJ, Evans CJ, Farquhar M, et al. Integrating Comprehensive Geriatric Assessment for people with COPD and frailty starting pulmonary rehabilitation: the Breathe Plus feasibility trial protocol. ERJ Open Res 2021; 7: 00717-2020 [https://doi.org/10.1183/23120541.007172020].

This study is registered at www.isrctn.com with identifier number ISRCTN13051922. Data will be available on request.

Received: 1 Oct 2020 | Accepted after revision: 21 Dec 2020

Copyright $\odot$ ERS 2021. This article is open access and distributed under the terms of the Creative Commons Attribution Non-Commercial Licence 4.0. 


\section{Introduction}

One in five people with COPD are living with frailty [1]. Frailty is broadly understood as a multidimensional syndrome characterised by decreases in reserve and diminished resistance to stressors [2]. People with both COPD and frailty are at increased risk of mortality [3,4] and have increased rates of hospitalisation [4] compared to non-frail counterparts. They experience poorer physical function, health status and quality of life $[4,5]$, and have increased anxiety and depression symptoms [6].

Pulmonary rehabilitation typically comprises twice-weekly, supervised, outpatient exercise sessions (involving progressive resistance and aerobic training based on individualised prescriptions) over 6-12 weeks, plus education to support self-management [7]. Importantly, pulmonary rehabilitation is equally, if not more, efficacious in people living with COPD and frailty: it can improve breathlessness, exercise performance, self-reported physical activity levels, anxiety and depression symptoms, and health status $[6,8]$, as well as reducing frailty $[6,9]$. However, people living with both COPD and frailty are less likely to start and complete pulmonary rehabilitation [6]. Developing and testing adapted approaches, encouraging engagement with pulmonary rehabilitation and improving outcomes for this population, are therefore high priorities.

To develop an adapted approach and our preliminary underpinning intervention theory, we conducted qualitative interviews with people living with both COPD and frailty referred for pulmonary rehabilitation [10]. Participants described continually striving to adapt to multidimensional losses associated with COPD and frailty, and variability in support received. While all were initially motivated to engage in pulmonary rehabilitation, changeable health and disruptions (e.g. exacerbations, worsening comorbidities, other appointments) could interfere with, and sometimes erode, their motivation and ability to attend. We then conducted a realist review to understand how exercise-based interventions for people with COPD might better address the context of frailty [11]. We found that successful exercise-based interventions for this group might need to foster trusting relationships with participants and a shared understanding of their priorities, individualise content to match priorities, have capacity to address multidimensional losses, and offer a flexible service delivery approach. Strategies to enable these mechanisms were identified, including a potential role for Comprehensive Geriatric Assessment (CGA).

CGA is a process incorporating a comprehensive, multidimensional review of a person's medical, psychological, functional and social capability, in order to develop individual recommendations and a care plan [12]. This typically involves treating any reversible causes, reviewing medicines including the impact of polypharmacy, providing nutritional support, cognitive assessment, and offering exercise training [12]. CGAs reduce functional dependency and mortality for older adults across inpatient [13] and outpatient settings [14]. In frail older adults, coordinated care based on CGA recommendations can improve quality of life and function, and reduce hospital admissions [15]. When introduced alongside other treatments, CGAs may increase capacity to benefit: pre-surgery CGAs can reduce post-operative complications and recovery [16] and CGAs delivered prior to chemotherapy are associated with increased tolerance and completion [17]. Recent work in inpatient respiratory rehabilitation has also suggested improved disease-specific health status and reduced exacerbations [18] following a CGA-directed approach.

Besides exercise, most core components of a CGA are not routinely addressed by outpatient pulmonary rehabilitation [7]. Integrating these two evidence-based interventions may therefore be of value to people living with both COPD and frailty. In our preliminary intervention theory (figure 1), we propose that addition of a CGA at the start of pulmonary rehabilitation may help foster some of the mechanisms deemed important by our development work [10, 11]. By fostering therapeutic alliance and tailored, multidimensional care recommendations, this approach may increase engagement with self-management and supportive services, including pulmonary rehabilitation, and contribute to improved health and function through increased reserves and adaptation.

Affiliations: ${ }^{1}$ King's College London, Cicely Saunders Institute of Palliative Care, Policy and Rehabilitation, London, UK. ${ }^{2}$ Sussex Community NHS Foundation Trust, Brighton General Hospital, Brighton, UK. ${ }^{3}$ University of East Anglia, School of Health Sciences, Norwich, UK. "King's College London, Clinical Trials Unit, London, UK. ${ }^{5}$ King's College London, Cicely Saunders Institute Public Involvement Group, London, UK. 'Guy's and St Thomas NHS Foundation Trust, Harefield Respiratory Research Group, London, UK. ${ }^{7}$ Imperial College, National Heart and Lung Institute, London, UK. ${ }^{8}$ King's College Hospital NHS Foundation Trust, Pulmonary Rehabilitation, London, UK. 'King's College Hospital NHS Foundation Trust, Dept of Clinical Gerontology, London, UK. ${ }^{10}$ King's College London, Dept of Twin Research and Genetic Epidemiology, London, UK. ${ }^{11}$ Guys and St. Thomas' NHS Foundation Trust, Dept of Ageing and Health, London, UK. ${ }^{12}$ Guy's and St Thomas NHS Foundation Trust, Harefield Pulmonary Rehabilitation Unit, London, UK.

Correspondence: Lisa Jane Brighton, King's College London, Cicely Saunders Institute of Palliative Care, Policy and Rehabilitation, Bessemer Road, London SE5 9PJ, UK. E-mail: lisa.brightondakcl.ac.uk 
Frailty context
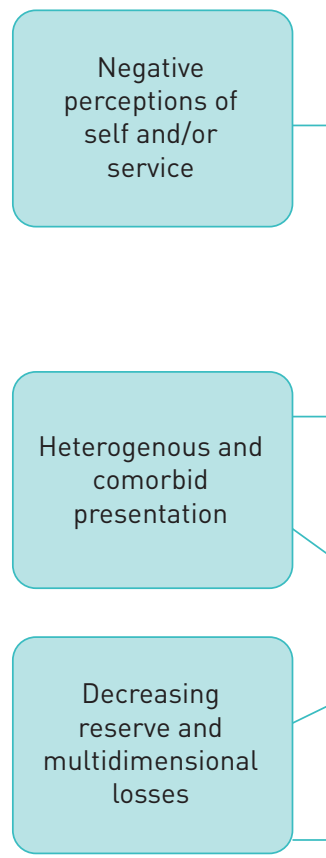

Intended mechanism

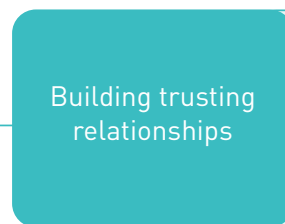

Shared

understanding of

priorities
Contributions of Comprehensive Geriatric Assessment

- Appointment with sufficient time to build a relationship

- Clinician skilled in empathetic communication

- Focused on working together to address health concerns
Outcomes

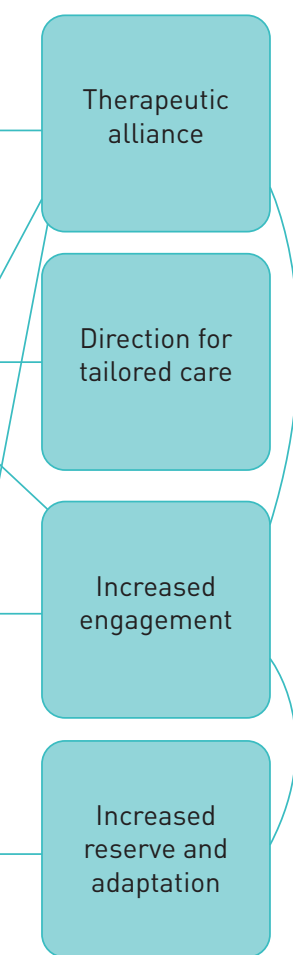

reserve and adaptation
Capacity to address

multidimensional losses
- Comprehensive assessment that attends to their priorities

- Shared decision making within appointment

- Recommendations shared with relevant providers
Individualised

content to match

priorities
- Priorities discussed and listened to

- Recommendations and follow-up individualised to the person

- Clinician skilled in managing multimorbidity and complexity

- Multidimensional assessment (e.g. physical, mental)

- Multidisciplinary representation within geriatric teams

- Strong cross-disciplinary relationships

FIGURE 1 Preliminary intervention theory of integrating a Comprehensive Geriatric Assessment for people with COPD and frailty starting pulmonary rehabilitation.

The proposed intervention is inherently complex: it includes multiple interacting components and relies on complex behaviours from participants and professionals [19]. Moreover, we will be working within complex systems, made up of individual, organisational and societal influences [20]. As such there are multiple uncertainties around the feasibility and acceptability of the intervention, and how best to evaluate its impact, that must be addressed prior to an effectiveness trial [20]. This study aims to determine the feasibility of conducting a randomised controlled trial of an integrated CGA for people with COPD and frailty starting pulmonary rehabilitation. Objectives are shown in table 1.

\section{Methods \\ Design}

The Breathe Plus feasibility trial will use a multicentre, mixed-method, randomised, controlled, assessor-blind trial design. Participants will be randomised 1:1 to two parallel groups: usual care or usual care plus a CGA. Quantitative intervention and trial data (including process data and participant outcome measures at baseline, 90 days and 180 days) will be collected alongside qualitative interview data from a subset of participants and staff (figure 2).

\section{TABLE 1 Study objectives}

1) To explore the acceptability of the intervention for participants and staff

2) To define, and understand the fidelity of, integrating a Comprehensive Geriatric Assessment, including how it differs from and impacts on usual care

3) To refine the intervention theory around integration of a Comprehensive Geriatric Assessment for this population

4) To estimate the appropriateness of the proposed eligibility criteria and study processes in successfully recruiting and retaining participants in the trial

5) To estimate risk of contamination between trial groups and unblinding in the trial

6) To explore the appropriateness and acceptability of proposed outcome measures and trial processes for participants and staff 


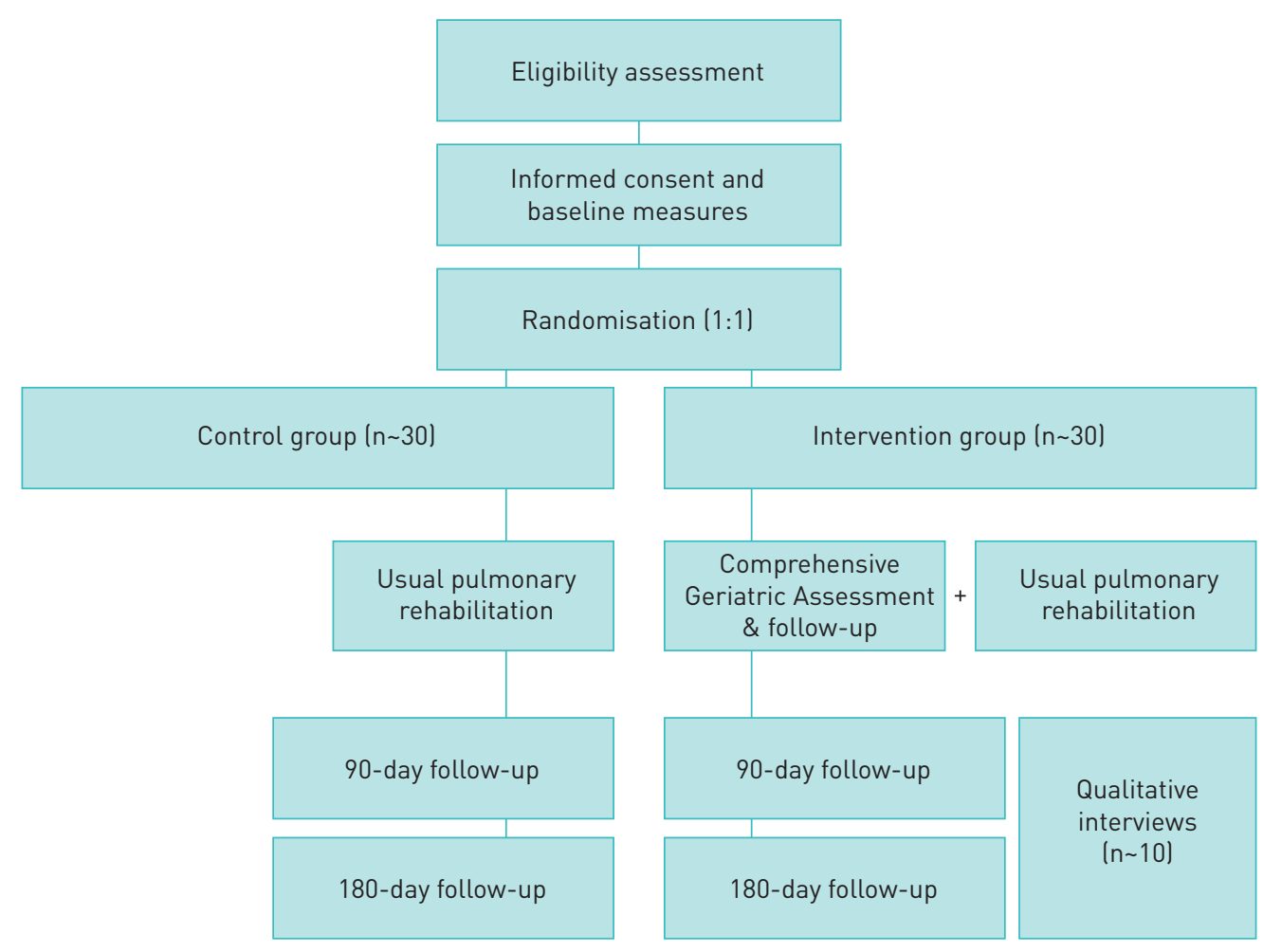

FIGURE 2 Overview of the Breathe Plus feasibility trial design.

\section{Setting}

Participants will be recruited from three outpatient pulmonary rehabilitation services based in hospitals in London, UK. Data collection will take place at participants' place of preference, typically: the hospitals, the university, participants' place of residence or via telephone.

\section{Participants}

Participants will include people living with both COPD and frailty referred for outpatient pulmonary rehabilitation. Full inclusion/exclusion criteria are shown in table 2.

The Clinical Frailty Scale is easy to administer in clinical settings and has been shown to be reliable and comparable to the Frailty Phenotype [23]. As the Clinical Frailty Scale should only be used after a formal clinical assessment [24] it will be incorporated alongside usual pre-pulmonary rehabilitation assessments. The research team will provide professionals using the Clinical Frailty Scale with training, drawing on published resources [25].

\section{TABLE 2 Inclusion and exclusion criteria}

\section{Inclusion criteria}

Adults aged 50 years or older

Physician diagnosis of chronic obstructive pulmonary disease lin line with GOLD criteria [21])

Referred for outpatient pulmonary rehabilitation (in line with BTS guidance [7])

Rockwood Clinical Frailty Scale score of $\geqslant 5$ [22]

\section{Exclusion criteria}

Lacking mental capacity to provide informed consent Unable to communicate verbally and respond to questions in written English and no interpreters to enable this

Receiving specialist geriatric services involving a geriatric doctor in previous or upcoming month

GOLD: Global initiative for chronic Obstructive Lung Disease; BTS: British Thoracic Society. \#: during the coronavirus disease 2019 pandemic, this will be inclusive of standard pulmonary rehabilitation available, including supported home-based exercise programmes. 
Sampling and recruitment

We will sample participants consecutively from people attending pre-pulmonary rehabilitation assessments. These assessments may take place remotely during the coronavirus pandemic. Pulmonary rehabilitation staff at each site will check people's eligibility during this assessment and introduce them to the study if applicable. Those interested in participating will receive a participant information sheet, and a researcher will discuss the study with them in detail. Those agreeing to participate will be asked to provide a record of consent. We anticipate recruitment of our target sample in 12 months across three sites.

Interventions

Usual care

All participants will continue their planned pulmonary rehabilitation course (typically at least twice-weekly supervised outpatient exercise sessions plus education for at least 6 weeks; may include amended remote-facilitated rehabilitation during the coronavirus pandemic). Usual care may also include interactions with specialist and non-specialist health and social care as required (e.g. respiratory consultant, general practitioner, other specialists that support comorbid conditions, emergency and/or hospital care, and care from integrated COPD nursing teams that support hospital discharge and management of exacerbations at home). All usual care contacts are permitted in both trial arms and will be recorded as feasibility trial data.

\section{Comprehensive Geriatric Assessment}

In addition to usual care, the intervention group will receive a CGA as soon as possible following completion of baseline measures, ideally prior to starting pulmonary rehabilitation. CGA is a process comprising a comprehensive assessment, development of a tailored care plan, and follow-up as required. We will encourage teams to work with their local materials (e.g. clinic proformas) to align intervention delivery with their usual practice.

The CGA will be led by a geriatric consultant. The initial appointment will typically be delivered as a one-to-one face-to-face appointment in an outpatient clinic, lasting approximately $1 \mathrm{~h}$ (during the coronavirus pandemic, remote delivery via video/phone may also be used). This will include a full medical assessment and history, and typically a review of functional and psychosocial issues, management of geriatric syndromes (e.g. frailty, falls, sarcopenia, incontinence, malnutrition, sensory impairment) and/or advance care planning, as relevant for the person. A resulting individualised care plan will be communicated back to the participant and relevant health care professionals (e.g. general practitioner, pulmonary rehabilitation team), for actioning. In all cases, tailoring of the Comprehensive Geriatric Assessment, subsequent follow-up and decision to discharge will be led by the geriatrician.

Support will be provided for travel where necessary. For two recruiting sites, the geriatric clinic is at the same hospital as the pre-pulmonary rehabilitation assessment. For one recruiting site, the geriatric clinic is at a different, nearby hospital.

\section{Feasibility outcomes and progression criteria}

Our primary feasibility outcomes relate to intervention fidelity and acceptability. Table 3 lists all feasibility outcomes, contributing data and progression criteria to full trial.

\section{Data collection}

\section{Service and trial process data}

Process data will be used to understand intervention delivery, potential impacts on usual care and trial design appropriateness. To describe CGA service delivery (intervention group participants only) we will collect: CGA date, recommendations, and follow-up, including (where applicable) when and who completed the recommendations. To describe pulmonary rehabilitation service delivery (all participants), we will collect: number of pulmonary rehabilitation sessions attended, adaptations made (e.g. dose reduction, delays in completion), and completion of post-pulmonary rehabilitation assessment. To describe the trial processes, we will collect: trial screening and recruitment rates, participation at each timepoint, mode of data collection, missing data, and unblinding.

\section{Participant characteristics and clinical outcomes}

Baseline demographic characteristics will be obtained through routinely collected data from the pulmonary rehabilitation teams, and self-reported questionnaires. These will include personal characteristics (age, sex, ethnicity), health and function (Forced expiratory volume in $1 \mathrm{~s}$ (FEV1) \% predicted, Medical Research Council (MRC) dyspnoea, exacerbations, 6-min walk test and/or incremental shuttle walk test, comorbidities, smoking status) and social factors (gender, living alone or with others, presence of an 
TABLE 3 Breathe Plus feasibility outcomes, contributing data and progression criteria

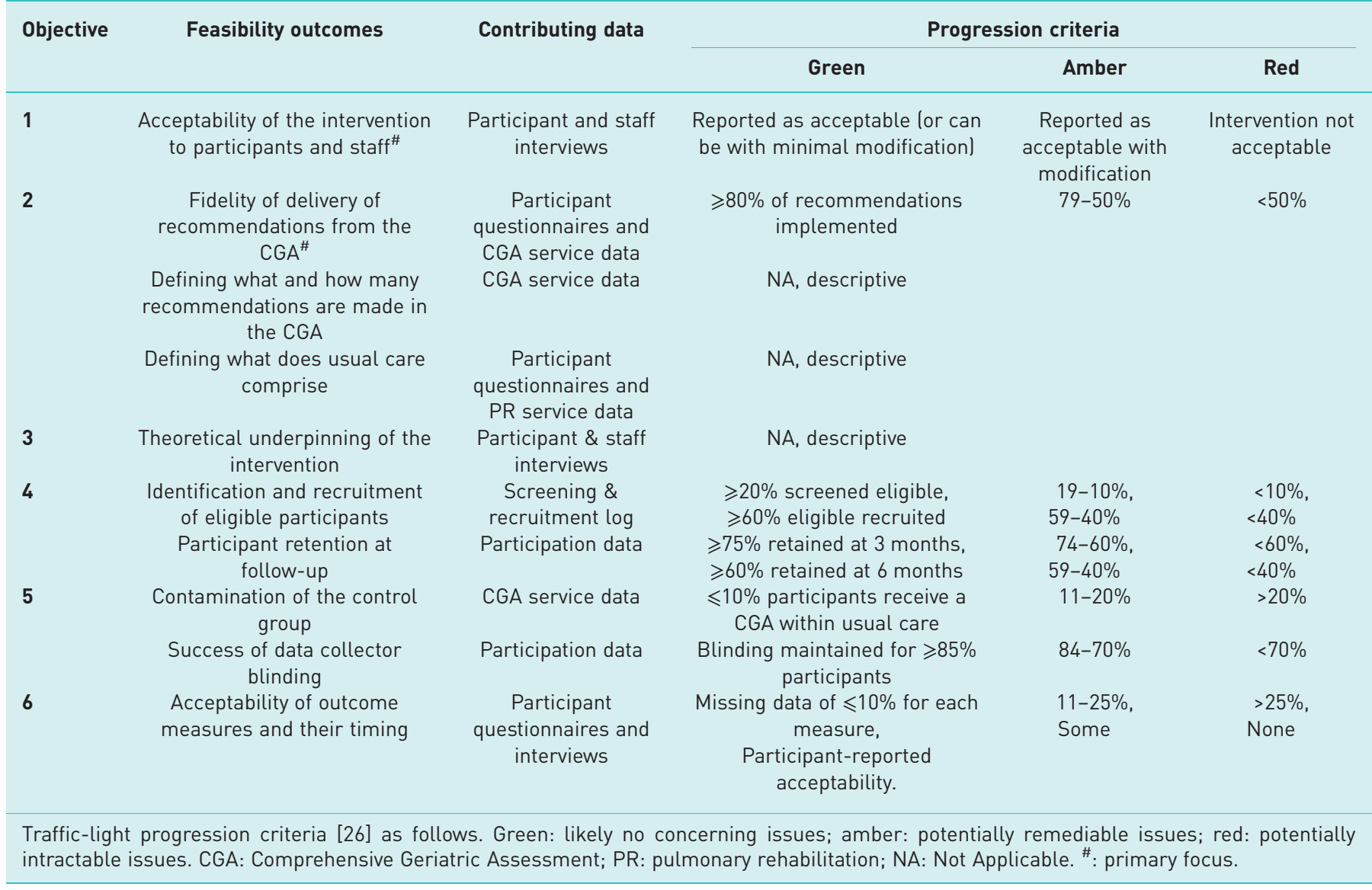

informal carer, being an informal carer, housing status, formal education level, English Indices of Social Deprivation).

The clinical outcome measures reflecting multiple domains (table 4) will be collected at baseline, 90 days and 180 days post-randomisation. In most cases, questionnaires will be completed with support from the researcher, but self-completion with return by post will also be allowed. While an in-person visit will be sought, all outcomes except physical frailty could be collected by phone if required. When completed by phone, participants will be sent a copy of the questionnaire to view during the call.

\section{Nested qualitative interviews}

Nested semi-structured qualitative interviews will be conducted with a sub-sample of participants and staff to address feasibility objectives 1 (intervention acceptability), 3 (theoretical underpinning) and 6 (outcome acceptability) (table 3).

We will interview approximately 10 intervention group participants following their second (90-day) data collection timepoint. Interviewing intervention participants only was deemed most appropriate as our primary feasibility objectives relate to intervention acceptability. Purposive sampling will be used to obtain maximum variation in relation to site and intervention fidelity, with consideration of diversity in terms of living status, outcomes, and questionnaire completion where possible. Informal carers will be welcome to participate alongside participants. Interviews will explore experiences of the intervention and trial participation.

We will also interview approximately five staff involved in the delivery of the trial. Purposive sampling will be used to obtain maximum variation in relation to site and team type (e.g. pulmonary rehabilitation, geriatrics). Interviews will explore experiences of the trial and perceptions of the intervention.

Interview schedules will be informed by the theoretical framework of acceptability [33] and reviewed by relevant stakeholders (people with relevant clinical, academic and/or personal experience). Interviews will 


\section{Domain}

\section{Physical frailty}

\section{Health-related quality of life}

\section{Activities of daily living}

Health status

\section{Anxiety and depression}

Loneliness

Service use

\section{Measure}

Short Physical Performance Battery (SPPB) [27]

Chronic Respiratory Questionnaire (CRQ-SR) [28]

Manchester Respiratory Activities of Daily Living questionnaire [29].

Euro-Qol 5D-5L

Hospital Anxiety and Depression Questionnaire (HADS) [30]

De Jong Gierveld Loneliness Scale (6-item) [31]

Client Service Receipt Inventory (CSRI) [32]

\section{Description}

Incorporates 4-m gait speed, five sit-to-stands, and static balance tests. It takes approximately 10-15 mins to complete, requires a floor mark for the gait-speed test, chair for sit-to-stand, and a timer, and results in a score from $0-12$ ( $\leqslant 7$ indicating frailty, 8-9 pre-frailty, and 10-12 robustness). This scale contains 20 items measuring the impact of chronic respiratory disease across four domains: dyspnoea, mastery, fatigue, and emotional function. Each item is scored from 1-7, and the mean score across each domain is calculated. Higher scores indicate better health status.

This measure includes 21 self-report items across four domains: mobility, kitchen activities, domestic tasks, and leisure activities. Most items are scored 0-1 based on responses of doing tasks "not at all", "with help", "alone with difficulty" or "alone easily" and total scores range from 0 to 21 , where 21 indicates no impairment in daily activities.

This measure contains five descriptive items (mobility, self-care, usual activities, pain, and anxiety/depression) with a five-point scale from "no problems" to "unable/extreme problems", and a visual analogue scale asking participants to rate their health from 0 (worst health imaginable) to 100 (best health imaginable). Descriptive item scores are converted into a single index value for health status, where a high value represents higher health status. Scores would also contribute to health economic analyses in a future effectiveness study.

This is a 14-item questionnaire with two subscales: anxiety (seven items) and depression (seven items). Items are scored on a scale of 0 to 3 . Items are summed creating a maximum score of 21 on each subscale, where higher scores indicate higher levels of symptoms of anxiety or depression. This shortened version includes three items measuring social loneliness, and three items measuring emotional loneliness, scored as 0 or 1 . When summed, a higher score indicates higher levels of loneliness.

This measure asks participants about their contacts with hospital and community health care services, any investigations or diagnostic tests, help from informal carers, and equipment used, over the past 3 months. This measure would also contribute to health economic analysis in a future effectiveness study.

be digitally audio-recorded. Field notes will record interview flow, contextual factors, participant responses and personal reflections. Stopping of recruitment will be based on sufficiency of information power [34] to answer the feasibility objectives.

\section{Sample size}

We intend to recruit a total of 60 participants (30 to the intervention group, 30 to the control group). This sample size was deemed to give an acceptable level of precision for our quantitative feasibility outcomes [35].

\section{Randomisation and allocation concealment}

Randomisation will occur as soon as possible following consent form completion and baseline assessment. Eligible participants will be randomly allocated 1:1 to the intervention or control group.

Randomisation method of minimisation was chosen with factors defined by site (1, 2 and 3 ), breathlessness (MRC dyspnoea $2-3$ and $4-5$ ), exacerbations ( $\geqslant 2$ and $<2$ in the past year) and living alone status (yes and no), along with an algorithm that contains an element of simple randomisation in order to preserve pre-randomisation allocation concealment.

The authorised researchers will randomise participants via the independent web-based randomisation system, run by the King's Clinical Trials Unit, which will automatically email the randomisation result to relevant members of the research team (e.g. chief investigator, research assistant) in a pre-specified blinded or unblinded format, depending on their role. An unblinded member of the research team will inform participants of their group allocation by phone. 


\section{Blinding}

It will not be possible to blind participants and intervention providers. Blinded researchers will collect follow-up clinical outcome assessments and complete the quantitative analysis.

\section{Analysis}

Quantitative data

Data will be entered by authorised researchers into a secure password-protected web-based Elsevier MACRO Electronic Data Capture system created in collaboration with the King's Clinical Trials Unit, which will capture a full audit trail of data entry and amendments. At trial end, following verification of data accuracy, the dataset will be locked for analysis.

A single intention-to-treat analysis will occur. Feasibility outcomes (table 3) will be described using proportions and corresponding 95\% confidence intervals, or, for continuous variables as mean $\pm \mathrm{SD}$ and median (interquartile range), depending on the data distribution. Where source data is not already quantified (e.g. CGA service notes), inductive content analysis will be used to summarise this information.

Baseline characteristics of the intervention and control participants will be summarised using descriptive statistics. Participant flow through the trial will be reported in a Consolidated Standards of Reporting Trials (CONSORT) flow diagram. In keeping with the feasibility aims, clinical outcomes will be described, but no inferential statistics used. Safety data will also be summarised.

\section{Qualitative data}

Qualitative interview data will be transcribed verbatim and link-anonymised. Framework analysis [36] will be used to explore the three qualitative feasibility outcomes (intervention acceptability, intervention theory and outcome acceptability; table 3).

The analysis process will begin with familiarisation with transcripts and field notes. An analytical framework will then be developed drawing deductively on the research objectives and relevant theoretical frameworks (including our preliminary intervention theory). The analytical framework will also be open to inductively generated participant-raised issues. This framework will be revised through revisiting the original data to ensure fair interpretation, engaging with wider relevant literature that may deepen our understanding, and review by relevant stakeholders to encourage a more nuanced reading. The analytical framework will then be systematically applied to the dataset, charting summarised data in a case-by-theme matrix. We will then map and interpret key findings and analytical themes in relation to the feasibility outcomes. The qualitative findings will be presented using a narrative approach, incorporating illustrative participant quotes. Although presented as a linear process, we will move forwards and backwards through these stages as thinking develops and changes [36]. Qualitative data will be mixed with the quantitative data during interpretation and reporting.

\section{Integration and recommendations for progression to a full trial}

Progression to the full trial will be determined based on feasibility trial results meeting pre-defined criteria (table 3) within the context of qualitative data [26]; in particular, where the qualitative data provides additional understanding and potential remedies for data falling in the amber/red zones. The data will be considered alongside views of key stakeholders including trial team members, clinicians involved in its delivery, and people affected by COPD and frailty. Conclusions and key recommendations in relation to each feasibility outcome will be reported with justifications, to aid transparency around the final decision.

\section{Monitoring adverse events}

All adverse events will be recorded in the study database and monitored for the duration of the trial. The study may be terminated if safety or ethical concerns are raised over the intervention and/or trial processes, or if there is a noticeable increase in number of deaths, emergency attendances or hospital admissions in either arm, as reviewed and recommended by joint trial management group/data monitoring committee at bi-annual meetings or ad hoc meetings (if necessary).

\section{Patient and public involvement}

Public involvement members affected by COPD and frailty, including members of our project team, and members of other relevant local public involvement groups (e.g. at the Cicely Saunders Institute, Harefield Breathing Group, and the local Biomedical Research Centre Respiratory group), have been involved in the intervention development work $[10,11]$ and continue to contribute to this feasibility trial. To date, we have received public involvement feedback on the trial name, recruitment processes, participant information materials, outcome measures and qualitative interview topic guides. Ongoing involvement includes discussing trial progress, troubleshooting arising challenges, assisting with the qualitative analysis, 
seeking their reflections and interpretations in relation to findings and advising on dissemination. At the end of the trial, we will use the Guidance for Reporting Involvement of Patients and the Public short form [37] to guide a critical reflection on the public involvement in the study.

\section{Ethics}

This study has been approved by the London City and East Research Ethics Committee (ref. 19/LO/1402). All participants provide a record of informed consent for their participation.

\section{Protocol version and amendments}

This paper reflects version 3.0 of the study protocol dated June 25, 2020. We have made two protocol amendments to date. Based on our experience with the first few participants, we allowed qualitative interviews to occur earlier (from 90 days onwards, rather than 180 days) to facilitate participation and recall. Due to restrictions on in-person contacts during the coronavirus disease 2019 pandemic, we have also expanded data collection, usual care and intervention procedures to be inclusive of remote methods.

\section{Dissemination}

A plain-English summary will be sent to participants opting to receive this. We will submit the results for publication in an open-access peer-reviewed journal, with authorship eligibility according to the International Committee of Medical Journal Editors criteria [38]. Findings will be reported following the CONSORT guidelines for pilot and feasibility trials [39] and Template for Intervention Description and Replication statement [40]. We will further share findings with clinical, academic and public stakeholders across websites, social media and presentations at local, national and international meetings. The full study protocol and anonymised data will be available on request.

\section{Discussion}

There is growing recognition of the potential for integration between geriatric and respiratory care [41, 42]. The Breathe Plus feasibility trial will test the feasibility of integrating two evidence-based approaches: Comprehensive Geriatric Assessment and pulmonary rehabilitation. We hope that this will both add value to rehabilitation services and better address frailty and its consequences in people with respiratory disease.

While bringing together two areas of effective respiratory and geriatric care seems a clear opportunity to improve outcomes for people with COPD and frailty, in practice, it requires integration of multiple complex systems. This preliminary work is therefore essential to address the uncertainties surrounding joining of established practices, potential benefits and burdens for participants and staff, and whether our proposed methods would be capable of capturing such impacts in a future effectiveness trial. It has already been necessary to add some flexibility in our methods due to coronavirus-related disruptions, and it may be that further adaptations to this changing context are required. However, by exploring the intervention theory alongside processes and outcomes, we hope we can explore the impact of such changes and inform further context-specific implementation should this approach be successful.

Acknowledgements: Thank you to all the patient, informal carer and public representatives who contributed to the study design, including project team members from the Harefield Breathing Group, and members of the BRC Respiratory PPI group, and the Cicely Saunders Institute public involvement group. We are grateful to the pulmonary rehabilitation and geriatric teams who are supporting this work, and to Rebecca Wilson (King's College London, London, UK) for her assistance with data management plans.

Support statement: This project is funded by a National Institute for Health Research (NIHR) Career Development Fellowship (CDF-2017-10-009) held by M. Maddocks. This research was supported by the NIHR Collaboration for Leadership in Applied Health Research and Care South London, now recommissioned as NIHR Applied Research Collaboration South London. C.J. Evans is funded by a Health Education England/NIHR Senior Clinical Lectureship (ICA-SCL-2015-01-001). C. Nolan is supported by a NIHR Research for Patient Benefit grant (PB-PG-0816-20022). This publication presents independent research funded by the National Institute for Health Research (NIHR). The views expressed in this publication are those of the author(s) and not necessarily those of the NHS, NIHR or the Department of Health and Social Care. Funding information for this article has been deposited with the Crossref Funder Registry.

Conflict of interest: L.J. Brighton has nothing to disclose. C.J. Evans has nothing to disclose. M. Farquhar has nothing to disclose. K. Bristowe has nothing to disclose. A. Kata has nothing to disclose. J. Higman has nothing to disclose M. Ogden has nothing to disclose. C. Nolan reports personal fees from Novartis outside the submitted work. D. Yi has nothing to disclose. W. Gao has nothing to disclose. M. Koulopoulou has nothing to disclose. S. Hasan has nothing to disclose. C. Steves has nothing to disclose. W.D-C. Man reports grants from the National Institute for Health Research and the British Lung Foundation, personal fees from Jazz Pharmaceuticals and Mundipharma, grants from Pfizer, personal fees from Novartis, and nonfinancial support from GSK, outside the submitted work. M. Maddocks has nothing to disclose. 


\section{References}

1 Marengoni A, Vetrano DL, Manes-Gravina E, et al. The relationship between COPD and frailty: a systematic review and meta-analysis of observational studies. Chest 2018; 154: 21-40.

2 Rodriguez-Manas L, Feart C, Mann G, et al. Searching for an operational definition of frailty: a Delphi method based consensus statement: the frailty operative definition-consensus conference project. J Gerontol A Biol Sci Med Sci 2013; 68: 62-67.

3 Galizia G, Cacciatore F, Testa G, et al. Role of clinical frailty on long-term mortality of elderly subjects with and without chronic obstructive pulmonary disease. Aging Clin Exp Res 2011; 23: 118-125.

4 Kennedy CC, Novotny PJ, LeBrasseur NK, et al. Frailty and clinical outcomes in chronic obstructive pulmonary disease. Ann Am Thorac Soc 2019; 16: 217-224.

5 Kusunose M, Oga T, Nakamura S, et al. Frailty and patient-reported outcomes in subjects with chronic obstructive pulmonary disease: are they independent entities? BMJ Open Respir Res 2017; 4: e000196.

6 Maddocks M, Kon SS, Canavan JL, et al. Physical frailty and pulmonary rehabilitation in COPD: a prospective cohort study. Thorax 2016; 71: 988-995.

7 British Thoracic Society. Quality standards for pulmonary rehabilitation in adults. Br Thorac Soc Rep 2014; 6 : $1-32$.

8 Blindenbach S, Vrancken JWFA, van der Zeijden $\mathrm{H}$, et al. [Effects of geriatric COPD rehabilitation on hospital admissions and exercise tolerance: a retrospective observational study]. Tijdshr Gerontol Geriatr 2017; 48: 112-120.

9 Mittal N, Raj R, Islam E, et al. Pulmonary rehabilitation improves frailty and gait speed in some ambulatory patients with chronic lung diseases. Southwest Respir Crit Care Chron 2015; 3: 2-10.

10 Brighton LJ, Bristowe K, Bayly J, et al. Experiences of pulmonary rehabilitation in people living with COPD and frailty: a qualitative interview study. Ann Am Thorac Soc 2020; 17: 1213-1221. e-pub before print

11 Brighton LJ, Evans CJ, Man W, et al. Improving exercise-based interventions for people living with both COPD and frailty: a realist review. Int J Chron Obstruct Pulmon Dis 2020; 15: 841-855.

12 Turner G, Clegg A. Best practice guidelines for the management of frailty: a British Geriatrics Society, Age UK and Royal College of General Practitioners report. Age Ageing 2014; 43: 744-747.

13 Ellis G, Whitehead MA, Robinson D, et al. Comprehensive Geriatric Assessment for older adults admitted to hospital: meta-analysis of randomised controlled trials. BMJ 2011; 343: d6553.

14 Reuben DB, Frank JC, Hirsch SH, et al. A randomized clinical trial of outpatient Comprehensive Geriatric Assessment coupled with an intervention to increase adherence to recommendations. J Am Geriatr Soc 1999; 47: 269-276.

15 Boult C, Green AF, Boult LB, et al. Successful models of comprehensive care for older adults with chronic conditions: evidence for the Institute of Medicine's "retooling for an aging America" report. J Am Geriatr Soc 2009; 57: 2328-2337.

16 Vidan M, Serra JA, Moreno C, et al. Efficacy of a comprehensive geriatric intervention in older patients hospitalized for hip fracture: a randomized, controlled trial. J Am Geriatr Soc 2005; 53: 1476-1482.

17 Kalsi T, Babic-Illman G, Ross PJ, et al. The impact of Comprehensive Geriatric Assessment interventions on tolerance to chemotherapy in older people. Br J Cancer 2015; 112: 1435-1444.

18 van Dam van Isselt EF, van Eijk M, van Geloven N, et al. A prospective cohort study on the effects of geriatric rehabilitation following acute exacerbations of COPD. J Am Med Dir Assoc 2019; 20: 850-856.e2.

19 Craig P, Dieppe P, Macintyre S, et al. Developing and evaluating complex interventions: the new Medical Research Council guidance. BMJ 2008; 337: a1655.

20 Fletcher A, Jamal F, Moore G, et al. Realist complex intervention science: Applying realist principles across all phases of the Medical Research Council framework for developing and evaluating complex interventions. Evaluation (Lond) 2016; 22: 286-303.

21 Global Initiative for Chronic Obstructive Lung Disease. Pocket guide to COPD diagnosis, management, and prevention: a guide for health care professionals. Retrieved from: www.goldcopd.org/wp-content/uploads/2020/03/ GOLD-2020-POCKET-GUIDE-ver1.0_FINAL-WMV.pdf Date last accessed: July 9, 2020; date last updated: November 2019.

22 Rockwood K, Song X, MacKnight C, et al. A global clinical measure of fitness and frailty in elderly people. CMAJ 2005; 173: 489-495.

23 Islam A, Muir-Hunter SW, Speechley M, et al. Facilitating frailty identification: comparison of two methods among community-dwelling order adults. J Frailty Aging 2014; 3: 216-221.

24 British Geriatrics Society. Recognising frailty. www.bgs.org.uk/resources/recognising-frailty Date last accessed: June 2019; date last updated: 11 June 2014.

25 The Ottawa Hospital. Clinical Frailty Scale (CFS) Training Module www.rise.articulate.com/share/ deb4rT02lvONbq4AfcMNRUudcd6QMts3\#/ Date last accessed: October 22, 2019; date last updated: 2019.

26 Avery KNL, Williamson PR, Gamble C, et al. Informing efficient randomised controlled trials: exploration of challenges in developing progression criteria for internal pilot studies. BMJ Open 2017; 7: e013537.

27 Guralnik JM, Simonsick EM, Ferrucci L, et al. A Short Physical Performance Battery Assessing Lower Extremity Function: Association With Self-Reported Disability and Prediction of Mortality and Nursing Home Admission. J Gerontol 1994; 49: M85-M94.

28 Williams JEA, Singh SJ, Sewell L, et al. Development of a self-reported Chronic Respiratory Questionnaire (CRQ-SR). Thorax 2001; 56: 954-959.

29 Yohannes AM, Roomi J, Winn S, et al. The Manchester Respiratory Activities of Daily Living questionnaire: development, reliability, validity, and responsiveness to pulmonary rehabilitation. J Am Geriatr Soc 2000; 48: 1496-1500.

30 Zigmond AS, Snaith RP. The hospital anxiety and depression scale. Acta Psychiatr Scand 1983; 67: 361-370.

31 Gierveld JDJ, Tilburg TV. A 6-item scale for overall, emotional, and social loneliness: Confirmatory tests on survey data. Res aging 2006; 28 : 582-598.

32 Beecham J, Knapp M. Costing psychiatric interventions. In: Thornicroft G, ed. Measuring mental health needs. 2nd edn. London, Gaskell, 2001; pp. 200-224.

33 Sekhon M, Cartwright M, Francis JJ. Acceptability of healthcare interventions: an overview of reviews and development of a theoretical framework. BMC Health Serv Res 2017; 17: 88. 
34 Malterud K, Siersma VD, Guassora AD. Sample size in qualitative interview studies: guided by information power. Qual Health Res 2016; 26: 1753-1760.

35 Hooper R. Justifying sample size for a feasibility study. Research Design Service. National Institute for Health Research London. www.rds-london.nihr.ac.uk/wpcms/wp-content/uploads/2019/02/Justifying-sample-size-forfeasibility-study-updated-22-Feb-2019.pdf Date last accessed: September 18, 2020; date last updated: 22 February 2019.

36 Ritchie J, Spencer L. Qualitative data analysis for applied policy research. In: Bryman A, Burgess B, eds. Analyzing Qualitative Data. 1st edn. Routledge, London, 1994; pp. 173-194.

37 Staniszewska S, Brett J, Simera I, et al. GRIPP2 reporting checklists: tools to improve reporting of patient and public involvement in research. BMJ 2017; 358: j3453.

38 International Committee of Medical Journal Editors. Defining the Role of Authors and Contributors. www.icmje. org/recommendations/browse/roles-and-responsibilities/defining-the-role-of-authors-and-contributors.html Date last accessed: July 232020.

39 Eldridge SM, Chan CL, Campbell MJ, et al. CONSORT 2010 statement: extension to randomised pilot and feasibility trials. Pilot Feasibility Stud 2016; 2: 64.

40 Hoffmann TC, Glasziou PP, Boutron I, et al. Better reporting of interventions: template for intervention description and replication (TIDieR) checklist and guide. BMJ 2014; 348: g1687.

41 Brighton LJ, Bone AE, Maddocks M. Supportive and palliative care for people with chronic respiratory disease and frailty. Curr Opin Support Palliat Care 2020; 14: 206-212.

42 Iyer AS, Curtis JR, Meier DE. Proactive integration of geriatrics and palliative care principles into practice for chronic obstructive pulmonary disease. JAMA Intern Med 2020; 180: 815-816. 